\title{
miR-202 suppresses prostate cancer growth and metastasis by targeting PIK3CA
}

\author{
SHENGPING ZHANG ${ }^{1 *}$, JIARONG CAI $^{2 *}$, WENJUN XIE $^{3}, \mathrm{HUI} \mathrm{LUO}^{3}$ and FEI YANG ${ }^{2}$ \\ ${ }^{1}$ Department of Urology, The People's Hospital of Longhua, Shenzhen, Guangdong 518109; Departments of ${ }^{2}$ Urology and \\ ${ }^{3}$ Operating Room, The Third Affiliated Hospital of Sun Yat-Sen University, Guangzhou, Guandong 510630, P.R. China
}

Received February 7, 2018; Accepted June 4, 2018

DOI: $10.3892 /$ etm.2018.6296

\begin{abstract}
MicroRNA (miR)-202 has been reported to be involved in the regulation of human cancer progression including bladder cancer, non-small cell lung cancer, pancreatic cancer and esophageal squamous cell carcinoma. However, the function of miR-202 in prostate cancer remains largely unknown. The present study demonstrated that miR-202 was downregulated in human prostate cancer tissues and cell lines. And overexpression of miR-202 significantly inhibited the proliferation, migration and invasion of prostate cancer cells, but induced cell apoptosis. Moreover, miR-202 suppressed tumor growth in vivo. Regarding the underlying mechanism, it was revealed that phosphatidylinositol-4,5-bisphosphate 3-kinase catalytic subunit $\alpha$ (PIK3CA) was a target gene of miR-202 in prostate cancer cells. Overexpression of miR-202 inhibited the mRNA and protein levels of PIK3CA in prostate cancer cells. Moreover, overexpression of PIK3CA abolished the inhibitory effects of miR-202 on prostate cancer cell proliferation, migration and invasion in vitro. Taken together, these findings demonstrated that miR-202 served as a tumor suppressor in prostate cancer by directly targeting PIK3CA.
\end{abstract}

\section{Introduction}

As one of the most common and aggressive cancers in male, prostate cancer (PC) contributes to a great number of cancer-related mortality around the world $(1,2)$. Although surgery combined with chemotherapy seems to be effective for treatment of localized PC, most diagnosed PC patients have metastases because of no effective diagnostic biomarkers $(3,4)$. Due to the distant metastasis of $\mathrm{PC}$, the 5-year survival rate of $\mathrm{PC}$ remains

Correspondence to: Dr Fei Yang, Department of Urology, The Third Affiliated Hospital of Sun Yat-Sen University, 600 Tianhe Road, Tianhe, Guangzhou, Guandong 510630, P.R. China

E-mail: feiyang188a@126.com

*Contributed equally

Key words: miR-202, prostate cancer, proliferation, migration, PIK3CA unsatisfactory (5). The incidence of PC is still quickly increasing and PC remains an important health problem worldwide (6). Therefore, it is urgently required to determine the molecular mechanism of PC development and progression.

MicroRNAs (miRNAs) are about 20 nucleotides in length and regulate the degradation of target mRNAs by associating with the 3'-URT region of mRNAs (7). miRNAs have been proven to regulate all kinds of biological processes, such as development, proliferation, apoptosis and metastasis (8-10). Dysregulation of miRNA expression is often observed in human cancers, including PC (11). For example, miR-141 suppresses PC stem cells and metastasis by targeting a cohort of pro-metastasis genes (12). miR-194 suppresses PC cell migration and invasion by downregulating human nuclear distribution protein (13). miR-141-3p promotes PC cell proliferation through inhibiting kruppel-like factor-9 expression (14). Downregulated miR-26a modulates PC cell proliferation and apoptosis by targeting COX-2 (15). miRNAs have been widely demonstrated to be important regulators in PC. Therefore, investigating the regulatory mechanism of miRNAs will benefit for the treatment of PC.

Emerging evidence shows that miR-202 acts as a tumor suppressor in some tumors. For instance, Yang et al (16) reported that miR-202 inhibits cell proliferation, migration and invasion of glioma by directly targeting metadherin. Meng et al (17), shown that miR-202 promotes cell apoptosis in esophageal squamous cell carcinoma. However, the role of miR-202 in PC is largely unknown. In this study, we found that the expression of miR-202 was significantly downregulated in PC tissues and cell lines. And overexpression of miR-202 markedly inhibited the proliferation, migration and invasion of PC cells. Moreover, miR-202 overexpression promoted the apoptosis of PC cells. Mechanistically, we found that phosphatidylinositol-4,5-bisphosphate 3-kinase catalytic subunit $\alpha$ (PIK3CA) is the target gene of miR-202 in PC cells. Overexpression of miR-202 inhibited the mRNA and protein levels of PIK3CA. Finally, we found that restoration of PIK3CA rescued the proliferation, migration and invasion of miR-202-overexpressing PC cells. Taken together, our results indicated that miR-202 acts as a tumor suppressor in PC by targeting PIK3CA.

\section{Materials and methods}

Patient samples. A total of 54 PC tissue specimens and 11 adjacent normal prostate tissues were obtained from primary 
PC patients in the Third Affiliated Hospital of Sun Yat-Sen University. The resected tissues were immediately frozen in liquid nitrogen and stored at $-80^{\circ} \mathrm{C}$ before use. All patients agreed and signed a written informed consent for tissue donation for research purposes before tissue collection. The study was approved by the Institutional Human Experiment and Ethic Committee of the Third Affiliated Hospital of Sun Yat-Sen University.

Cell lines and cell culture. Human PC cell lines including LNCaP, 22Rv1, PC-3 and DU145 and normal prostate epithelial cells (RWPE-1) were purchased from Cell Bank of Chinese Academy of Sciences (Shanghai, China). PC cells were grown in RPMI 1640 (Gibco; Thermo Fisher Scientific, Inc., Waltham, MA, USA) supplemented with $10 \%$ fetal bovine serum (FBS; Gibco; Thermo Fisher Scientific, Inc.), $100 \mathrm{U} / \mathrm{ml}$ Penicillin and $0.1 \mu \mathrm{g} / \mathrm{ml}$ Streptomycin. RWPE-1 cells were cultured in keratinocyte-serum free medium (Gibco; Thermo Fisher Scientific, Inc.) containing human recombinant epidermal growth factor ( $5 \mathrm{ng} / \mathrm{ml}$; Thermo Fisher Scientific, Inc.) and bovine pituitary extract $(0.05 \mathrm{mg} / \mathrm{ml}$; Thermo Fisher Scientific, Inc.). Cells were cultured in a humidified atmosphere of $5 \% \mathrm{CO}_{2}$ at $37^{\circ} \mathrm{C}$.

Xenograft tumor formation. Six-week-old BALB/c male nude mice were obtained from Shanghai Laboratory Animal Center (Chinese Academy of Sciences, Shanghai, China). Tumor-bearing mice were maintained under specific pathogen-free conditions in Experimental Animal Center of the Third Affiliated Hospital of Sun Yat-Sen University. All animal experiments were performed in accordance with the Guide for Care and Use of Laboratory Animal and all experimental protocols were approved by the Animal Ethics Committee of the Third Affiliated Hospital of Sun Yat-Sen University. For the subcutaneous xenograft mouse model, DU145 cells $\left(5 \times 10^{6}\right)$ that were transiently transfected with miR-202 mimics or miR-NC were suspended in $100 \mu$ medium and subcutaneously injected into the left and right backside flanks of nude mice ( $n=6$ mice/group) respectively. The volume of the in vivo tumor xenograft was calculated. Five weeks after initial injection, mice were sacrificed and PC xenograft was weighed.

Cell proliferation. Cell proliferation was measured by Cell Counting Kit-8 (CCK-8; Sigma-Aldrich; Merck KGaA, Darmstadt, Germany). Briefly, cells were seeded into a 96-well plate at $1 \times 10^{4}$ cells/well and cultured for $48 \mathrm{~h}$ after transfection. Cells were then treated with CCK- 8 reagent $(10 \mu \mathrm{l} /$ well $)$ at $37^{\circ} \mathrm{C}$ for $4 \mathrm{~h}$. Absorbance at $450 \mathrm{~nm}$ was detected using a microplate reader (BioTek Instruments, Inc., Winooski, VT, USA).

Flow cytometric analysis of apoptosis. After double staining with FITC-Annexin $\mathrm{V}$ and propidium iodide (PI) using Annexin V-FITC apoptosis detection kit (BD Biosciences, Franklin Lakes, NJ, USA) at room temperature, cells were analyzed with a flow cytometry (BD LSRFortessa flow cytometer; BD Biosciences) equipped with CellQuest software (BD Biosciences).

Transwell assay. Cells were suspended in serum-free medium, and $3 \times 10^{4}$ cells $(100 \mu \mathrm{l})$ was plated into the upper chambers of
Transwell inserts (8.0-mm pore size; Corning Incorporated, Corning, NY, USA) for invasion (with Matrigel) or migration (without Matrigel) assays. The inserts were placed in 24-well plates containing $600 \mu \mathrm{l}$ media with $10 \%$ FBS. After incubation for $24 \mathrm{~h}$ for migration and $48 \mathrm{~h}$ for invasion, cells were fixed for $20 \mathrm{~min}$ and stained with $1 \%$ crystal violet. The cell numbers were calculated and imaged under a microscope from five random fields.

Real-time quantitative PCR. Total RNA from PC tissues and cells were extracted using Trizol (Invitrogen; Thermo Fisher Scientific, Inc.). For miRNA expression, RT reactions were performed with a One Step PrimeScript miRNA cDNA Synthesis kit, followed by PCR with $\mathrm{SYBR}^{\circledR}$ Premix Ex Taq (Takara Biotechnology Co., Ltd, Dalian, China). For mRNA, cDNA was synthesized from the total RNA using a PrimeScript RT Reagent Kit (TTakara Biotechnology Co., Ltd., Dalian, China). qPCR amplification reactions were performed with SYBR $^{\circledR}$ Premix Ex Taq II with ROX (Takara Biotechnology Co., Ltd.). U6 or GAPDH was used for normalization and all data were calculated by the $2^{-\Delta \Delta \mathrm{Cq}}$ method (18).

Statistical analysis. Data are presented as mean \pm SD and analyzed using GraphPad Prism 5 (GraphPad Software Inc., San Diego, USA) and SPSS v.21.0 software (IBM Corp., Armonk, NY, USA). Statistical significance was investigated through a two-tailed, unpaired Student's t-test, one-way ANOVA followed by Tukey's post hoc test or a chi-square test as appropriate. $\mathrm{P}<0.05$ was considered to indicate a statistically significant difference.

\section{Results}

miR-202 is downregulated in PC tissues and cells. To determine the role of miR-202 in PC, we used qRT-PCR to analyze the expression of miR-202 in PC tissues. We found that miR-202 was significantly downregulated in PC tissues compared to adjacent normal tissues (Fig. 1A). Then we checked the expression of miR-202 in 11 paired PC tissues. And we found that most PC tissues showed lower expression of miR-202 than paired normal tissues (Fig. 1B). We also examined the expression of miR-202 in PC cell lines. We found that miR-202 was downregulated in PC cell lines (LNCaP, 22Rv1, PC-3 and DU145 cells) compared to normal RWPE-1 (Fig. 1C).

miR-202 overexpression inhibits cell proliferation, migration and invasion of $P C$. To further explore the function of miR-202, we overexpressed miR-202 in DU145 and LNCaP cells by transfection with miR-202 mimics (Fig. 2A). Then we performed CCK8 assay to check the proliferation of DU145 and LNCaP cells. We found that overexpression of miR-202 significantly inhibited the proliferatioin of DU145 and LNCaP cells (Fig. 2B). Notably, we also analyzed the effect of miR-202 overexpression in RWPE-1 cell growth, migration and invasion. However, the results were not significant compared to control group (data not shown). Moreover, by staining with AnnexinV/PI, we found that miR-202 overexpression markedly increased the apoptotic cells (Fig. 2C). PC metastasis greatly contributes to the malignance. Therefore, we evaluated the effect of miR-202 on cellular migration and invasion with 

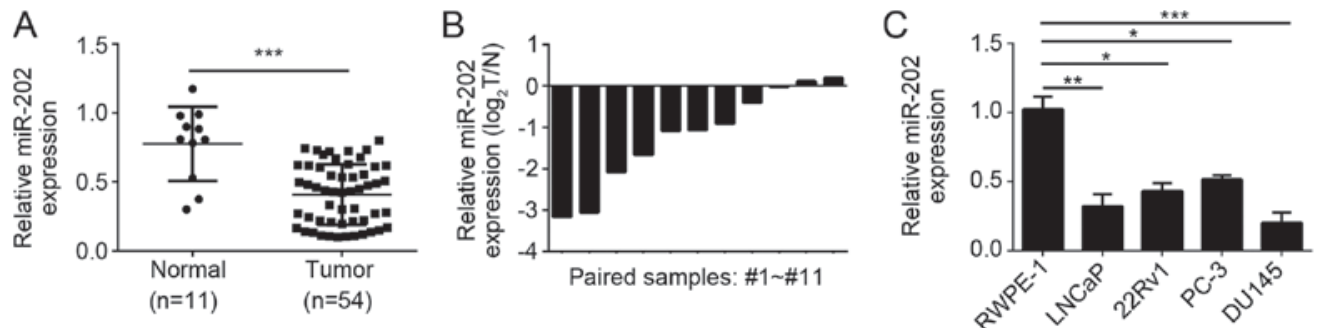

Figure 1. miR-202 was downregulated in prostate cancer tissues and cells. (A) qRT-PCR analysis indicated that miR-202 was significantly downregulated in prostate cancer tissues $(n=54)$ compared to the normal prostate tissues $(n=11)$. (B) qRT-PCR analysis of miR-202 levels in eleven pairs of prostate cancer tissues and matched adjacent normal prostate tissues. (C) qRT-PCR analysis of miR-202 levels in primary prostate epithelial cell lines and prostate cancer cells. ${ }^{*} \mathrm{P}<0.05,{ }^{* *} \mathrm{P}<0.01$ and ${ }^{* * * *} \mathrm{P}<0.001$ vs. the control group.
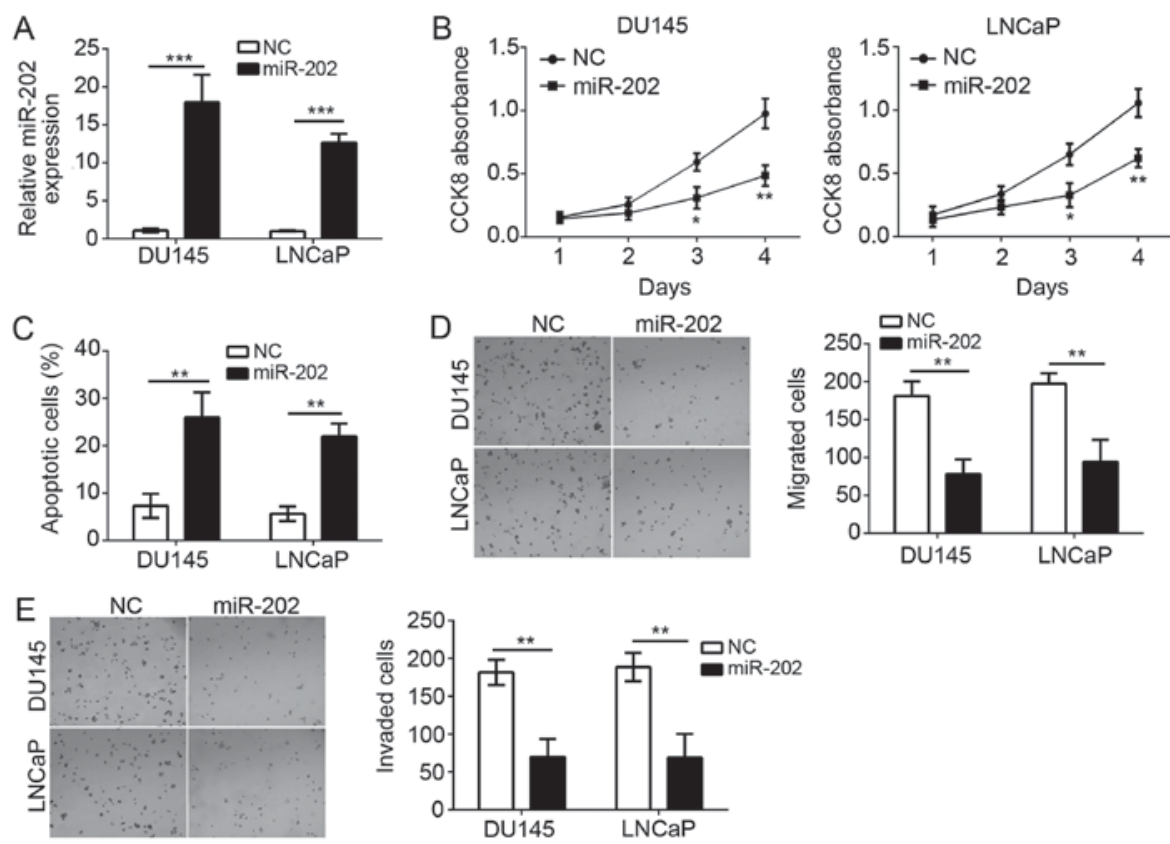

Figure 2. miR-202 overexpression inhibited cell proliferation, migration and invasion of prostate cancer. (A) qRT-PCR assay of miR-202 level in DU145 and LNCaP cells transfected miR-202 mimic or negative control (NC). (B) CCK8 assay of the proliferation of indicated prostate cells transfected with miR-202 mimic or negative control. (C) Cell apoptotic rate in DU145 and LNCaP cells was detected by FACS. (D and E) Cell migration and invasion were analyzed by transwell assay in DU145 and LNCaP cells. Magnification, $\mathrm{x} 100 .{ }^{*} \mathrm{P}<0.05,{ }^{* *} \mathrm{P}<0.01$ and ${ }^{* * * *} \mathrm{P}<0.01$ vs. the control group.

transwell assay. As shown in Fig. 2D-F, we found that overexpression of miR-202 significantly suppressed the numbers of migrated and invaded cells. Taken together, our findings indicated that miR-202 suppressed that proliferation, migration and invasion of PC cells but induced cellular apoptosis.

miR-202 overexpression suppresses tumor growth in vivo. To further investigate the function of miR-202 in vivo, we performed a xenograft experiment by subcutaneously injecting DU145 cells into the dorsal flank of nude mice. Tumor growth was closely monitored for 5 weeks. Results showed that overexpression of miR-202 significantly inhibited the tumor growth in vivo (Fig. 3A). Similarly, the tumor weight was also significantly decreased by overexpression of miR-202 (Fig. 3B and C). Notably, the expression of miR-202 was still upregulated compared to control tumor tissues while PIK3CA expression was significantly downregulated (Fig. 3D). Then we evaluated the cell proliferation of tumor tissues by western blot. We found that tumor tissues derived from miR-202-overexpressing DU145 cells showed less proliferation as the expression of Cyclin D1 and PCNA was lower than that in control group (Fig. 3E). Taken together, our findings demonstrated that overexpression of miR-202 inhibited tumor growth in vivo.

PIK3CA is a direct target of $m i R$-202. It has been widely acknowledged that miRNAs target the 3'-UTR region of specific mRNAs to exert essential biological functions. To explore miR-202-mediated mechanism, we made a computer-based sequence analysis by TargetScan website and identified PIK3CA as a potential target gene of miR-202. There was a possible binding site of miR-202 in the 3'-UTR region of PIK3CA (Fig. 4A). To determine whether miR-202 could target the mRNA of PIK3CA, we constructed luciferase reporter plasmid and performed luciferase activity reporter assay. As shown, overexpression of miR-202 significantly inhibited the luciferase activity in DU145 cells (Fig. 4B). And mutation of the binding site in the 3'-UTR region of PIK3CA abrogated the inhibition of luciferase activity by miR-202 (Fig. 4B). To illustrate that the endogenous miR-202 could regulate the expression of PIK3CA, we overexpressed miR-202 in DU145 cells and found 

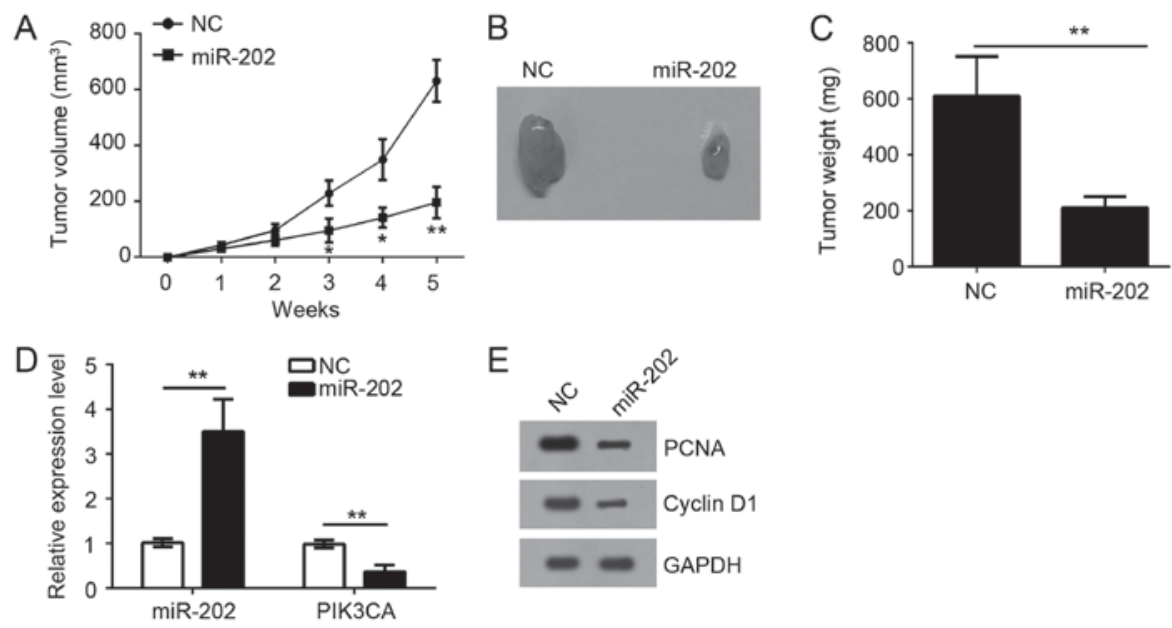

Figure 3. miR-202 overexpression suppressed tumor growth in vivo. (A) The growth curves were plotted to monitor tumor volumes for 5 weeks. (B) Representative images of the xenografts from respective groups of nude mice (n=6 mice/group). (C) Tumors were excised and weighed at the end of the experiment. (D) Relative expression of miR-202 and PIK3CA in formed tumor tissues by qRT-PCR. (E) The protein levels of cyclin D1 and PCNA were checked by western blot in formed tumor tissues. ${ }^{*} \mathrm{P}<0.05$ and ${ }^{* * *} \mathrm{P}<0.01$ vs. the control group. PIK3CA, phosphatidylinositol-4,5-bisphosphate 3-kinase catalytic subunit $\alpha$.

A

PIK3CA-WT: 5'-GAUUGCAUAGGAA-3'

miR-202:3'-UAUACGUAUCCUU-5

PIK3CA-Mut:5'-GAUACGUAUCCUU-3'

$\mathrm{D}$

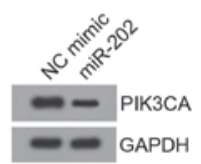

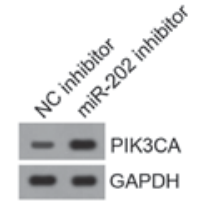
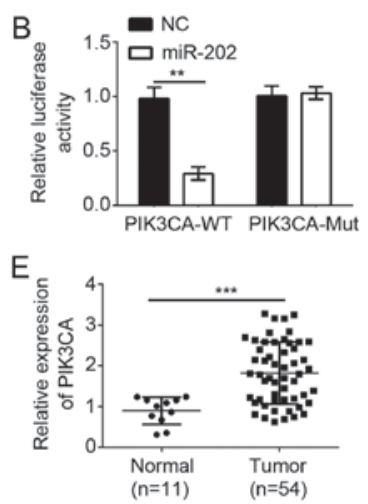
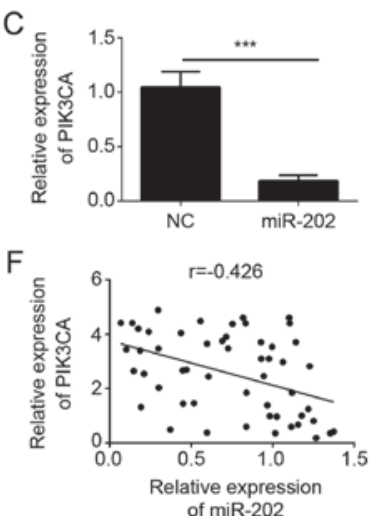

Figure 4. PIK3CA was a direct target of miR-202. (A) The seed sequences for miR-202 in the 3'-UTR of PIK3CA revealed by TargetScan analysis. (B) Luciferase reporter assay was performed $48 \mathrm{~h}$ after co-transfection in DU145 cells with WT-PIK3CA or Mut-PIK3CA vectors together with miR-202 or negative control. (C) qRT-PCR revealed the effects of miR-202 mimics on the expression level of PIK3CA mRNA. (D) Western blot analysis revealed the effects of miR-202 mimics on the expression level of PIK3CA protein. (E) Relative expression of PIK3CA in prostate cancer tissues and adjacent normal tissues by qRT-PCR. (F) Expression correlation between miR-202 and PIK3CA in prostate cancer tissues. ${ }^{* *} \mathrm{P}<0.01$ and ${ }^{* * *} \mathrm{P}<0.001$ vs. the control group. PIK3CA, phosphatidylinositol-4,5-bisphosphate 3-kinase catalytic subunit $\alpha$.

that its overexpression significantly inhibited the mRNA and protein levels of PIK3CA in PC cells (Fig. 4C and D). Besides, we found that PIK3CA expression was significantly upregulated in PC tissues compared to adjacent normal tissues (Fig. 4E) and inversely correlated with that of miR-202 (Fig. 4F), suggesting PIK3CA was a target of miR-202.

PIK3CA is involved in miR-202-mediated regulation of PC cell proliferation, apoptosis, migration and invasion. To determine whether miR-202 inhibited PC cell proliferation, migration and invasion by targeting PIK3CA, we performed a rescue assay. We restored the expression of PIK3CA in miR-202-overexpressed DU145 cells by transfection with PIK3CA ectopically expressing plasmid (Fig. 5A). Moreover, the effects of miR-202 overexpression on cellular proliferation, apoptosis, migration and invasion were significantly reversed by PIK3CA overexpression (Fig. 5B-E). These results indicated that miR-202 suppressor the development and progression of PC by downregulation of PIK3CA.

\section{Discussion}

$\mathrm{PC}$ is one of the most common and aggressive cancers in male and contributes to a great number of cancer-related mortality around the world $(1,2)$. Nowadays, the mechanism of PC development still remains elusive. And PC is still a major challenge for people's health. Therefore, it is of vital importance to explore the molecular mechanism contributing to PC progression. In this study, we explored the function of miR-202 and its mechanism involved in PC.

miRNAs have been widely acknowledged as important regulators in human cancers by serving as oncogenes or tumor suppressors $(19,20)$. miR-202 was also proven to regulate the occurrence of some tumors. For instance, Sun et al (21) reported that miR-202 suppresses proliferation and induces apoptosis of osteosarcoma cells by downregulating Gli2. Zhang et al (22), showed that miR-202 suppresses cell proliferation in human hepatocellular carcinoma by downregulating LRP6 post-transcriptionally. Wang et al (23), reported 
A
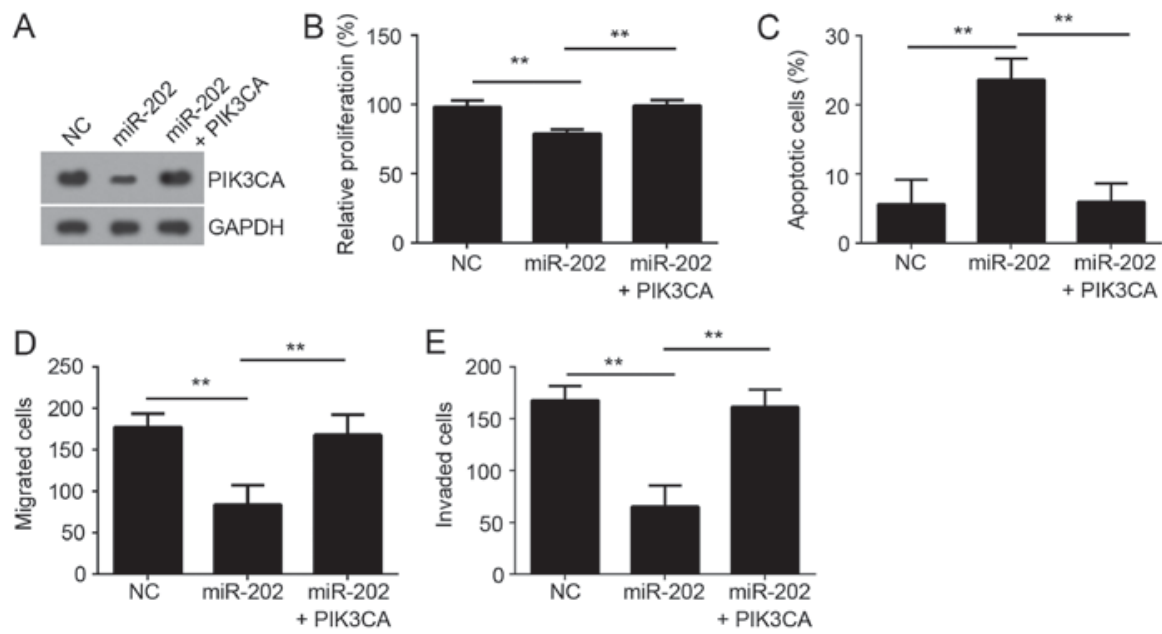

Figure 5. PIK3CA was involved in miR-202-mediated regulation of prostate cancer proliferation, apoptosis, migration and invasion. (A) Western blot assay of PIK3CA levels in DU145 cells cotransfected with miR-202 mimics and PIK3CA-overexpressing plasmid. (B) Cell proliferation was assessed by CCK-8. (C) Cell apoptosis was measured by FACS. (D) Cell migration and (E) invasion was detected by the transwell assay. ${ }^{* *} \mathrm{P}<0.01$ vs. the control group. PIK3CA, phosphatidylinositol-4,5-bisphosphate 3-kinase catalytic subunit $\alpha$.

that miR-202-3p inhibits cell proliferation by targeting ADP-ribosylation factor-like $5 \mathrm{~A}$ in human colorectal carcinoma. Additionally, Zhao et al (24) identified miR-202-3p was downregulated in gastric cancer and acted as a novel tumor suppressor. Nevertheless, the physiological function of miR-202 remains largely unknown in PC. In the present study, we found that the expression of miR-202 was downregulated in PC tissues and cell lines by qRT-PCR. Moreover, through CCK8, FACS and transwell assays, we found that overexpression of miR-202 significantly inhibited the proliferation, migration and invasion but induced apoptosis in PC. Moreover, xenograft experiments indicated that miR-202 suppressed tumor growth in vivo. Collectively, our results demonstrated that up-regulation of miR-202 in PC cells significantly inhibits cell proliferation, migration and invasion, which is consistent with previous reports about the function of miR-202 in other cancers.

PIK3CA is an essential subunit of phosphatidylinositol 3 -kinase (PI3K), which regulates the activation of AKT $(25,26)$. $\mathrm{PI} 3 \mathrm{~K} / \mathrm{AKT}$ pathway is a vital regulator in cell proliferation, metastasis and apoptosis by modulating downstream targets, such as mammalian target of rapamycin (mTOR) and Cyclin-dependent kinase inhibitors (27). Increasing evidence shows that PI3K/AKT pathway is closely related with the tumorigenesis, such as breast cancer (28), hepatocellular carcinoma (29) and PC (30). Dysregulated expression of PIK3CA led to abnormal activation of PI3K/AKP pathway and consequently development of cancers. In prostate, many studies reported that PIK3CA expression is linked to the progression of PC. For example, Agell et al (31) reported that PI3K signaling pathway is activated by PIK3CA mRNA overexpression and copy gain in prostate tumors. However, how miRNA regulates the expression of PIK3CA in PC remains elusive. In the present study, we found that PIK3CA was a direct target of miR-202 by bioinformatics prediction. Luciferase reporter demonstrated their interaction. Besides, we showed overexpression of miR-202 significantly inhibited the mRNA and protein levels of PIK3CA in PC cells. What's more, through functional experiments, we found that overexpression of PIK3CA significantly rescued the inhibitory effects of miR-202 on cellular proliferation, migration and invasion, and reduced cellular apoptosis.

In summary, our results demonstrated that miR-202 served as a tumor suppressor by targeting PIK3CA in PC, which provided a new sight on the development of therapeutic targets for PC treatment.

\section{Acknowledgements}

Not applicable.

\section{Funding}

The present study was supported by grants from key laboratory of Longhua, Shenzhen (grant no. 20150925A0410013). The authors declare no competing financial interests.

\section{Availability of data and materials}

All data generated or analyzed during the present study are included in this published article.

\section{Authors' contributions}

SZ, JC and FY initiated, designed this work, analyzed, interpreted the results and wrote this manuscript. WX and HL performed certain experiments. All authors read and approved the final manuscript.

\section{Ethics approval and consent to participate}

For the use of human samples, the protocol for this study was approved by the Institutional Ethics Committee of The People's hospital of Longhua and all enrolled patients signed a written informed consent document. In addition, all procedures involving animals conformed to the national guidelines of and were approved by the Animal Care Ethics Committee of The People's hospital of Longhua. 


\section{Patient consent for publication}

All patients within this study provide consent for the publication of their data.

\section{Competing interests}

The authors declare that they have no competing interests.

\section{References}

1. Okato A, Goto Y, Kurozumi A, Kato M, Kojima S, Matsushita R, Yonemori M, Miyamoto K, Ichikawa T and Seki N: Direct regulation of LAMP1 by tumor-suppressive microRNA-320a in prostate cancer. Int J Oncol 49: 111-122, 2016.

2. Jemal A, Bray F, Center MM, Ferlay J, Ward E and Forman D: Global cancer statistics. CA Cancer J Clin 61: 69-90, 2011.

3. Coleman RE: Clinical features of metastatic bone disease and risk of skeletal morbidity. Clin Cancer Res 12: 6243s-6249s, 2006.

4. Edlind MP and Hsieh AC: PI3K-AKT-mTOR signaling in prostate cancer progression and androgen deprivation therapy resistance. Asian J Androl 16: 378-386, 2014

5. Watson PA, Arora VK and Sawyers CL: Emerging mechanisms of resistance to androgen receptor inhibitors in prostate cancer. Nat Rev Cancer 15: 701-711, 2015.

6. Hu Y, Zhao Q, Rao J, Deng H, Yuan H and Xu B: Longitudinal trends in prostate cancer incidence, mortality, and survival of patients from two Shanghai city districts: A retrospective population-based cohort study, 2000-2009. BMC Public Health 14: 356, 2014.

7. Bartel DP: MicroRNAs: Genomics, biogenesis, mechanism, and function. Cell 116: 281-297, 2004.

8. Khan S, Brougham CL, Wall D, Newell J, Kerin MJ and Dwyer RM: Mir-339-5p acts as A tumour suppressor in breast cancer, mediated at least in part through regulation of cell proliferation. Ir J Med Sci 182: S343, 2013.

9. Wang T, Ren Y, Liu R, Ma J, Shi Y, Zhang L and Bu R miR-195-5p suppresses the proliferation, migration and invasion of oral squamous cell carcinoma by targeting TRIM14. Biomed Res Int 2017: 7378148, 2017.

10. Alipour A,Mojdehfarahbakhsh A, Tavakolian A, Morshedzadeh T, Asadi M, Mehdizadeh A and Nami M: Neural communication through theta-gamma cross-frequency coupling in a bistable motion perception task. J Integra Neurosci 15: 539-551, 2016.

11. Wang D, Lu G, Shao Y and Xu D: microRNA-802 inhibits epithelial-mesenchymal transition through targeting flotillin-2 in human prostate cancer. Biosci Rep 37: pii: BSR20160521, 2017.

12. Liu C, Liu R, Zhang D, Deng Q, Liu B, Chao HP, Rycaj K, Takata Y, Lin K, Lu Y, et al: MicroRNA-141 suppresses prostate cancer stem cells and metastasis by targeting a cohort of pro-metastasis genes. Nat Commun 8: 14270, 2017.

13. Kong Q, Chen XS, Tian T, Xia XY and Xu P: MicroRNA-194 suppresses prostate cancer migration and invasion by downregulating human nuclear distribution protein. Oncol Rep 37: 803-812, 2017.

14. Li JZ, Li J, Wang HQ, Li X, Wen B and Wang YJ: MiR-141-3p promotes prostate cancer cell proliferation through inhibiting kruppel-like factor-9 expression. Biochem Biophys Res Commun 482: 1381-1386, 2017.
15. Zhang J, Liang J and Huang J: Downregulated microRNA-26a modulates prostate cancer cell proliferation and apoptosis by targeting COX-2. Oncol Lett 12: 3397-3402, 2016.

16. Yang J, Fan B, Zhao Y and Fang J: MicroRNA-202 inhibits cell proliferation, migration and invasion of glioma by directly targeting metadherin. Oncol Rep 38: 1670-1678, 2017.

17. Meng X, Chen X, Lu P, Ma W, Yue D, Song L and Fan Q: miR-202 promotes cell apoptosis in esophageal squamous cell carcinoma by targeting HSF2. Oncol Res 25: 215-223, 2017.

18. Livak KJ and Schmittgen TD: Analysis of relative gene expression data using real-time quantitative PCR and the 2(-Delta Delta C(T)) method. Methods 25: 402-408, 2001

19. Damavandi Z, Torkashvand S, Vasei M, Soltani BM, Tavallaei M and Mowla SJ: Aberrant expression of breast development-related MicroRNAs, miR-22, miR-132, and miR-212, in breast tumor tissues. J Breast Cancer 19: 148-155, 2016.

20. Han SY, Han HB, Tian XY, Sun H, Xue D, Zhao C, Jiang ST, He XR, Zheng WX, Wang J, et al: MicroRNA-33a-3p suppresses cell migration and invasion by directly targeting $\mathrm{PBX} 3$ in human hepatocellular carcinoma. Oncotarget 7: 42461-42473, 2016.

21. Sun Z, Zhang T, Hong H, Liu Q and Zhang H: miR-202 suppresses proliferation and induces apoptosis of osteosarcoma cells by downregulating Gli2. Mol Cell Biochem 397: 277-283, 2014.

22. Zhang Y, Zheng DY, Xiong Y, Xue C, Chen G, Yan B and Ye Q: miR-202 suppresses cell proliferation in human hepatocellular carcinoma by downregulating LRP6 post-transcriptionally. FEBS Lett 588: 1913-1920, 2014

23. Wang Q, Huang Z, Guo W, Ni S, Xiao X, Wang L, Huang D, Tan C, Xu Q, Zha R, et al: microRNA-202-3p inhibits cell proliferation by targeting ADP-ribosylation factor-like 5A in human colorectal carcinoma. Clin Cancer Res 20: 1146-1157, 2014.

24. Zhao Y, Li CL, Wang M, Su L, Qu Y, Li J, Yu B, Yan M, Yu Y, Liu B and Zhu Z: Decrease of miR-202-3p expression, a novel tumor suppressor, in gastric cancer. PLoS One 8: e69756, 2013.

25. Chang F, Lee JT, Navolanic PM, Steelman LS, Shelton JG, Blalock WL, Franklin RA and McCubrey JA: Involvement of PI3K/Akt pathway in cell cycle progression, apoptosis, and neoplastic transformation: A target for cancer chemotherapy. Leukemia 17: 590-603, 2003.

26. Osaki M, Oshimura M and Ito H: PI3K-Akt pathway: Its functions and alterations in human cancer. Apoptosis 9: 667-676, 2004.

27. Nicholson KM and Anderson NG: The protein kinase B/Akt signalling pathway in human malignancy. Cell Signal 14: 381-395, 2002.

28. Woo SU, Sangai T, Akcakanat A, Chen H, Wei C and Meric-Bernstam F: Vertical inhibition of the PI3K/Akt/mTOR pathway is synergistic in breast cancer. Oncogenesis 6: e385, 2017.

29. Chai R, Fu L, Zheng Z, Liu T, Ji S and Li G: Resveratrol inhibits proliferation and migration through SIRT1 mediated post-translational modification of PI3K/AKT signaling in hepatocellular carcinoma cells. Mol Med Report 16: 8037-8044, 2017.

30. Chen R, Li Y, Buttyan R and Dong X: Implications of PI3K/AKT inhibition on REST protein stability and neuroendocrine phenotype acquisition in prostate cancer cells. Oncotarget 8: 84863-84876, 2017.

31. Agell L, Hernández S, Salido M, de Muga S, Juanpere N, Arumí-Uria M, Menendez S, Lorenzo M, Lorente JA, Serrano S and Lloreta $\mathrm{J}$ : PI3K signaling pathway is activated by PIK3CA mRNA overexpression and copy gain in prostate tumors, but PIK3CA, BRAF, KRAS and AKT1 mutations are infrequent events. Mod Pathol 24: 443-452, 2011. 